\title{
Comparison of the biological characteristics of human mesenchymal stem cells derived from exfoliated deciduous teeth, bone marrow, gingival tissue, and umbilical cord
}

\author{
JING LI $^{1}$, SHI-QING XU ${ }^{2}$, YU-MING ZHAO ${ }^{1}$, SHI YU ${ }^{1}$, LI-HONG GE ${ }^{1 *}$ and BAO-HUA XU ${ }^{3 *}$ \\ ${ }^{1}$ Department of Pediatric Dentistry, Peking University School and Hospital of Stomatology, Beijing 100081; \\ ${ }^{2}$ Institute of Clinical Medical Sciences; ${ }^{3}$ Dental Medical Center, China-Japan Friendship Hospital, Beijing 100029, P.R. China
}

Received January 26, 2018; Accepted August 30, 2018

DOI: $10.3892 / \mathrm{mmr} .2018 .9501$

\begin{abstract}
Different sources of mesenchymal stem cells (MSCs) may differ in their biological characteristics, which are important for their clinical application. In the present study, MSCs were isolated from human exfoliated deciduous teeth (SHED), bone marrow, gingival tissue and umbilical cord tissue, and their biological characteristics including surface markers, proliferation capacity, tumorigenicity and immunogenicity were analyzed by flow cytometric analysis, ELISA and co-culture with human lymphocytes, respectively. The results indicated that all four types of stem cells obtained from different sources expressed MSC surface markers, and they did not show tumorigenicity either in vivo or in vitro. Stem cells from SHED exhibited the strongest proliferation capacity. Umbilical cord-derived MSCs displayed the strongest
\end{abstract}

Correspondence to: Dr Li-Hong Ge, Department of Pediatric Dentistry, Peking University School and Hospital of Stomatology, 22 Zhongguancun Avenue South, Haidian, Beijing 100081, P.R. China

E-mail: gelihong0919@163.com

Dr Bao-Hua Xu, Dental Medical Center, China-Japan Friendship Hospital, 2 East Cherry Street, Chaoyang, Beijing 100029, P.R. China E-mail: zrkqxbh@163.com

${ }^{*}$ Contributed equally

Abbreviations: MSCs, mesenchymal stem cells; BMSCs, bone marrow mesenchymal stem cells; SHED, stem cells from human exfoliated deciduous teeth; UCMSCs, umbilical cord-derived mesenchymal stem cells; GMSCs, gingiva-derived mesenchymal stem cells; $\alpha$-MEM, $\alpha$-modified Eagle's minimum essential medium; FBS, fetal bovine serum; CK19, cytokeratin 19; EDTA, ethylenediaminetetraacetic acid; PBMCs, peripheral blood mononuclear cells; ELISA, enzyme-linked immunosorbent assay; PHA, phytohemagglutinin; HLA, human leukocyte antigen; IFN- $\gamma$, interferon- $\gamma$

Key words: human mesenchymal stem cells, cell proliferation, immunogenicity, tumorigenicity immunomodulatory ability, while bone marrow MSCs exhibited the best antigen-presenting potential in response to interferon- $\gamma$ stimulation. These results provide information on MSCs derived from different tissues, which may be helpful in their clinical application.

\section{Introduction}

Mesenchymal stem cells (MSCs) are derived from the early developmental mesoderm, and they are characterized as undifferentiated cells. They are considered to play important roles in development, postnatal growth, repair, regeneration, and homeostasis. These cells have attracted much interest regarding their possible clinical applications because of their self-renewing capacity and multi-lineage differentiation potential (1). MSCs can be used as ideal seed cells for the repair of injury to tissue and organs. In addition, these cells may also have immunomodulatory benefits in the treatment of autoimmune diseases and systemic diseases (2-4). Several investigations have shown the efficacy of MSCs in regenerative medicine both in vitro and in vivo (5-7). Although MSCs have been obtained from bone marrow, umbilical cord, and various odontogenic tissues, differences in their biological characteristics have not been fully clarified.

MSCs obtained from human bone marrow (BMSCs) have been intensively studied since their discovery. They have been demonstrated to have strong self-renewal capacity and can differentiate into a variety of tissue cells in a specific environment. They have been used widely in animal experimental models and clinical therapies for hematological system diseases, cardiovascular diseases, nervous system diseases, and osteogenesis-related disorders (8-12).

Stem cells from human exfoliated deciduous teeth (SHED) have been isolated from naturally exfoliated deciduous teeth with the capacity to differentiate into osteogenic and odontogenic cells, adipocytes, and neural cells. SHEDs are derived from a very accessible tissue resource and are capable of providing enough cells for potential clinical application via their high proliferation rate and expression of telomerase (13).

Umbilical cord-derived mesenchymal stem cells (UCMSCs) collected from umbilical cord tissues of healthy full-term babies have excellent proliferation potential and 
are free of ethical issues (14). UCMSCs have morphological, phenotypic, and multilineage differentiation potential similar to that of BMSCs; they are able to differentiate into functional hepatocyte-like cells in vitro, but their immunogenicity remains low (15).

Human gingival tissue from the oral cavity can often be easily obtained as a discarded biological sample without any morbidity or scar formation. Gingiva-derived mesenchymal stem cells (GMSCs) can be easily isolated from the gingival tissue, can be expanded easily in vitro, and possess multipotent differentiation potential and anti-inflammatory properties $(16,17)$. GMSCs are capable of regenerating bone defects, and they may be potentially useful in the reconstruction and regeneration of bone defects (18).

Besides their function in tissue reconstruction and regeneration, the low immunogenicity and immunoregulatory potential of MSCs are advantageous for their clinical application. It has been reported that BMSCs do not express major histocompatibility complex (MHC) class II molecules or costimulatory molecules required for T-cell activation, which are responsible for transplant rejection (19-21). UCMSCs can maintain low immunogenicity when they differentiate into other tissue cells (15); SHEDs show superior immunomodulatory effects compared to BMSCs (22), and GMSCs have reproducible and powerful immunomodulatory functions (23).

MSCs can be used for the treatment of many diseases associated with defective tissue regeneration and immune regulation. However, it is difficult to decide which type of stem cell should be used in clinical treatment according to the safety and immunogenicity of the cells. Therefore, we need to understand the biological characteristics of MSCs from different sources and the differences in their tumorigenicity and immunogenicity. BMSCs from alveolar bone and GMSCs can be obtained from discarded tissues during dental surgery, and obtaining the samples is less invasive to the donors. SHEDs and UCMSCs are obtained from discarded medical tissues, and are easy to obtain, non-invasive to donors, and plentiful. In this study, we compared the cell proliferation ability, tumorigenicity, and immunogenicity of BMSCs, SHEDs, UCMSCs, and GMSCs. The results provide information that is helpful for the clinical application of these cells.

\section{Materials and methods}

Isolation and culture of human MSCs. SHEDs, GMSCs, and UCMSCs were donated by the Oral Stem Cell Bank of Beijing, Tason Biotech Co., Ltd. (Beijing, China), each stem cell derived from three different individuals. Alveolar bone marrow samples were obtained from the mandibular bone of healthy patients (from 1 male and 2 female donors, aged 30, 28, and 20 years) after obtaining written informed consent. Sample collection was approved by the Institutional Review Board of Peking University School and Hospital of Stomatology (Beijing, China; no. PKUSSIRB-201734036). BMSCs were isolated from mandibular alveolar bone marrow. Cultures of all four types of MSCs were maintained in $\alpha$-modified Eagle's minimum essential medium ( $\alpha$-MEM; Gibco; Thermo Fisher Scientific, Inc., Waltham, MA, USA) supplemented with $10 \%$ fetal bovine serum (FBS; Biological Industries, Madison,
WI, USA) in $5 \% \mathrm{CO}_{2}$ at $37^{\circ} \mathrm{C}$. The cells were used in experiments after three to five passages, and for each experiment, all MSCs had the same passage number.

Biological characteristics of MSCs. Cells in the logarithmic growth phase were detached with $0.1 \%$ trypsin/0.1\% EDTA to produce a single-cell suspension. The cells were labeled with rabbit antihuman nestin (Merck KGaA, Darmstadt, Germany), vimentin, NANOG, and CD90 antibodies, mouse antihuman CD105 (all Abcam, Cambridge, MA, USA) and CD34 (BD Pharmingen, San Diego, CA, USA) antibodies, and goat antihuman cytokeratin19 (CK19; Santa Cruz Biotechnology, Santa Cruz, CA, USA) antibody, respectively, and approximately $1 \times 10^{6}$ cells were used for the detection of each molecule. Alexa Fluor 488-conjugated antirabbit, antimouse, and antigoat IgGs were used as the secondary antibodies. The labeled cells were thoroughly washed by centrifugation 3 times, resuspended in PBS solution, and flow cytometry (Cytomics Flow Cytometer EPICS XL; Beckman Coulter, Fullerton, CA, USA) was used to detect the fluorescence intensity and positive rate.

The self-renewal capacity of the MSCs was evaluated by colony-forming efficiency assays. $1 \times 10^{2}$ cells at passage 3 were seeded in 6 -well plates at $37^{\circ} \mathrm{C}$ with $5 \% \mathrm{CO}_{2}$ for 14 days. Then the cells were stained with $0.5 \%$ crystal violet (Sigma-Aldrich; Merck $\mathrm{KGaA}$ ) for $5 \mathrm{~min}$ at room temperature. Stained colonies with $>50$ cells were counted. Colony-forming efficiency was calculated as colony-forming unit numbers.

MSCs were induced by osteogenic, adipogenic, and chondrogenic differentiation kits (Biowit Technologies; Shenzhen, China) according to the manufacturer's protocol. $2 \times 10^{5}$ cells at passage 3 were seeded in 24 -well plates at $37^{\circ} \mathrm{C}$ with $5 \% \mathrm{CO}_{2}$. When the cells became $70-80 \%$ confluent, the medium was replaced by differentiation induction medium for 2-3 weeks. The cells were analyzed for osteogenesis, adipogenesis, and chondrogenesis by Alizarin Red staining, Oil Red O staining, and Alcian Blue staining.

Analysis of cell proliferation capacity. MSCs were seeded into 96-well plates at a density of $3 \times 10^{3}$ cells/well and then cultured for 7 days. Cell counting kit-8 (CCK-8; Dojindo, Kumamoto, Kyushu, Japan) solution was added to each well of the plate and the absorbance was measured at $450 \mathrm{~nm}$ every $24 \mathrm{~h}$ according to the manufacturer's protocol.

Tumorigenicity assay. To evaluate tumorigenicity in vitro, anchorage-independent growth was assessed with soft agar colony formation assay using a 6-well plate. The base agar layer was prepared from a $0.6 \%$ soft agar solution containing $\alpha$-MEM with $10 \%$ FBS. Then, $1 \times 10^{4}$ cells were suspended in $\alpha$-MEM containing $10 \%$ FBS and $0.35 \%$ agar solution and plated onto the base layer. HeLa cells were used as positive control. Plates were incubated at $37^{\circ} \mathrm{C}$ with $5 \% \mathrm{CO}_{2}$ for 21 days, after which colony formation was observed under a microscope.

The animal study was approved by the Animal Ethics Committee of China-Japan Friendship Hospital (Beijing, China; no. 170103). To examine tumorigenicity in vivo, 8-week-old $\mathrm{Nu} / \mathrm{Nu}$ male mice were purchased from Beijing Vital River Laboratory Animal Technology Company [quality certificate: SCXK (Beijing) 2012-0001]. All animals were 


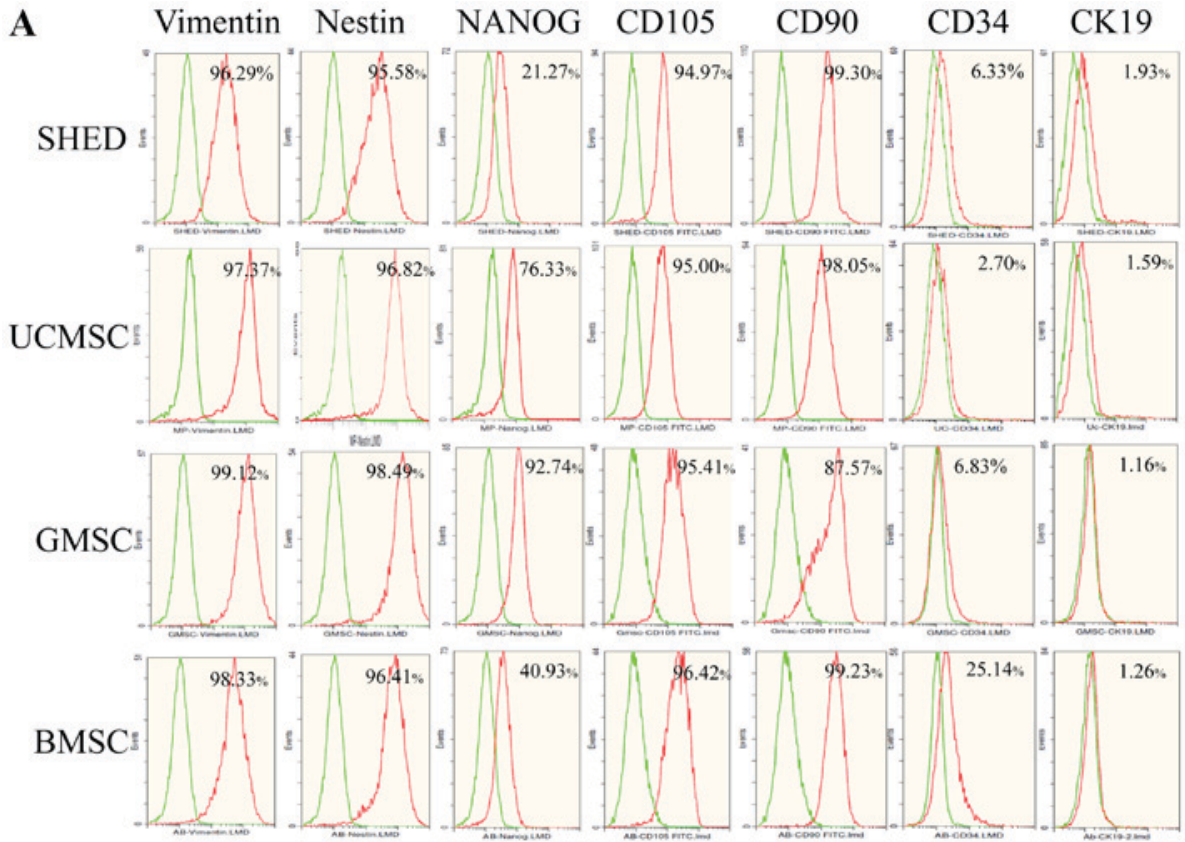

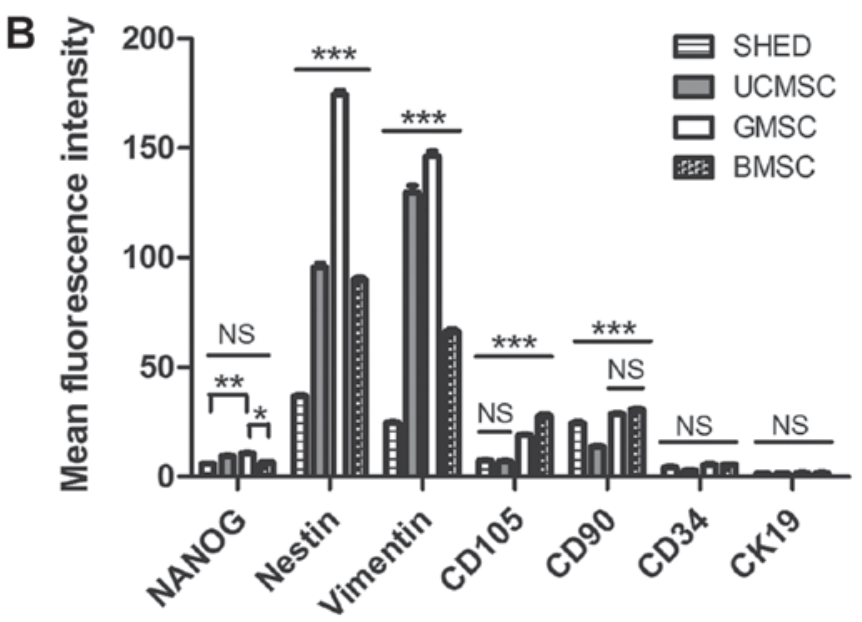

housed in the specific pathogen-free (SPF) facility [quality certificate: SYXK (Beijing) 2010-0011] in the Institute of Clinical Medical Sciences of China-Japan Friendship Hospital, and rats were maintained on a 12-h light:12-h dark cycle with free access to rodent chow and water. Mice were randomly divided into four groups of six mice each. All four types of MSCs were suspended in $\alpha$-MEM medium at a density of $2 \times 10^{6}$ cells $/ 100 \mu 1$, respectively. In each group, six nude mice were anesthetized via $2 \%$ isoflurane inhalation, and $2 \times 10^{6}$ MSCs were subcutaneously inoculated into the backs of each mouse. The grafts were observed for 6 months; twice daily for clinical signs in the mouse and twice weekly for the presence of a tumor. Tumor volume was calculated according to the following formula: $\mathrm{V}\left(\mathrm{mm}^{3}\right)=\left(\right.$ width $^{2} \mathrm{x}$ length $) / 2$.

At passage 4 of all four types of MSCs, karyotypes were analyzed respectively. $0.4 \mu \mathrm{g} / \mathrm{ml}$ colchicine (Gibco; Thermo Fisher Scientific, Inc.) was added to $1 \times 10^{6}$ cells for $4 \mathrm{~h}$. After centrifugation, hypotonic treatment, and then fixed. The substrate was dripped onto a glass slide, dried at $80^{\circ} \mathrm{C}$ for $2 \mathrm{~h}$, then digested with $0.5 \%$ trypsin for $10 \mathrm{sec}$, stained with $10 \%$ Giemsa, and observed and analyzed under a microscope.
Figure 1. (A) Surface marker expression of MSCs. (B) All four types of MSCs were positive for vimentin, nestin, NANOG, CD105 and CD90, and were negative for CD34 and CK19. Results represent the mean \pm standard deviation of three independent experiments. ${ }^{*} \mathrm{P}<0.05,{ }^{* *} \mathrm{P}<0.01$ and ${ }^{* * * *} \mathrm{P}<0.001$, as indicated. MSCs, mesenchymal stem cells; $\mathrm{CD}$, cluster of differentiation; SHEDs, stem cells from human exfoliated deciduous teeth; UCMSCs, umbilical cord-derived mesenchymal stem cells; GMSCs, gingiva-derived mesenchymal stem cells; BMSCs, bone marrow mesenchymal stem cells; CK19, cytokeratin19; NS, not significant.

Chromosome analysis was carried out by applying a scatter plot of the natural distribution and G-bands according to the guidelines of the International System for Chromosome Nomenclature (ISCN).

\section{Immunogenicity assay}

Mix lymphocyte proliferation assay. Human peripheral blood mononuclear cells (PBMCs) were isolated from healthy donors [sample collection was approved by the Institutional Review Board of Peking University School and Hospital of Stomatology (PKUSSIRB-201311108) and written informed consent were obtained from all the donors] using human peripheral blood lymphocyte separation solution (Tianjin HaoYang Biological Manufacture Co., Ltd., Tianjin, China) and density gradient centrifugation. Cells were cultured in RPMI-1640 medium supplemented with 10\% FBS, 2 mM glutamine (Gibco; Thermo Fisher Scientific, Inc.), $100 \mathrm{U} / \mathrm{ml}$ penicillin, and $100 \mu \mathrm{g} / \mathrm{ml}$ streptomycin (North China Pharmaceutical Limited by Share Ltd., Shijiazhuang, China) at $37^{\circ} \mathrm{C}$ with $5 \% \mathrm{CO}_{2}$. The PBMCs were depleted of adherent cells overnight and further cultured in PBMC medium. The experimental cells were 


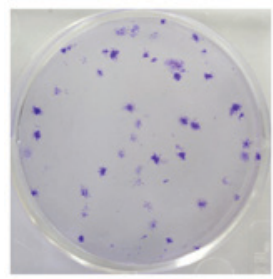

SHED

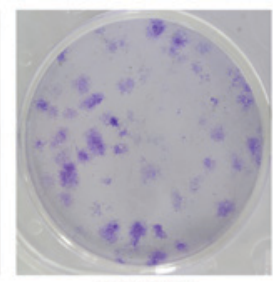

BMSC

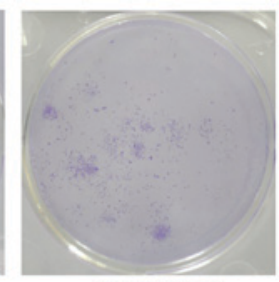

UCMSC

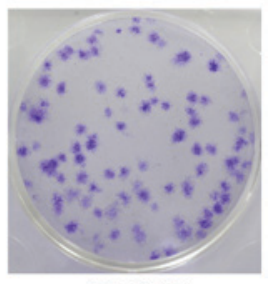

GMSC

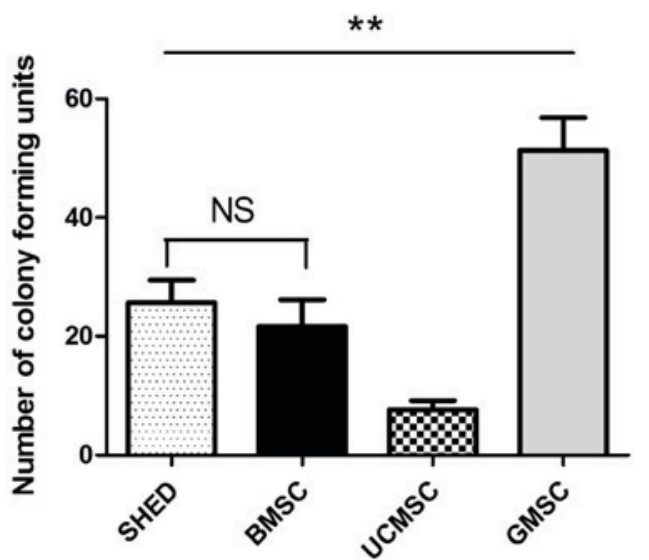

Figure 2. Colony-forming efficiency assay of MSCs. Colony formation was observed in all four MSCs cultured for 14 days. Colony-forming efficiency was different among the four MSCs. Results represent the mean \pm standard deviation of three independent experiments. ${ }^{* *} \mathrm{P}<0.01$, as indicated. NS, not significant; MSCs, mesenchymal stem cells; SHEDs, stem cells from human exfoliated deciduous teeth; UCMSCs, umbilical cord-derived mesenchymal stem cells; GMSCs, gingiva-derived mesenchymal stem cells; BMSCs, bone marrow mesenchymal stem cells.

divided into the following 6 groups: Control group: 1x10\%/well PBMCs were cultured in a 24 -well plate in normal medium as described above; positive control group: $10 \mu \mathrm{g} / \mathrm{ml}$ PHA (Sigma-Aldrich; Merck KGaA) with 1×10 ${ }^{6}$ PBMCs; and stem cell group: Each group of the four types of MSCs, 2x10 $/$ well, were co-cultured with $1 \times 10^{6}$ PBMCs, and the MSCs were treated with $25 \mu \mathrm{g} / \mathrm{ml}$ mitomycin C (Sigma-Aldrich; Merck $\mathrm{KGaA})$ at $37^{\circ} \mathrm{C}$ for $30 \mathrm{~min}$ before seeding into wells. Each group was co-cultured for $72 \mathrm{~h}$, the PBMCs were harvested and placed in 96-well plates, and CCK-8 was used to measure proliferation.

Detection of HLA-I, HLA-DR, CD80, and CD86. All four types of MSCs were detected by flow cytometry using fluorescein-isothiocyanate-conjugated or phycoerythrin-conjugated antibodies specific for HLA-I, HLA-DR (both Abcam), CD80, and CD86 (both R\&D Systems, Minneapolis, MN, USA) as previously described. To enhance the expression of immune related surface molecules, MSCs were pretreated with $100 \mathrm{U} / \mathrm{ml}$ IFN- $\gamma$ (interferon- $\gamma$; Peprotech, Rocky Hill, NJ, USA) for $72 \mathrm{~h}$ as previously described (24), and then the IFN- $\gamma$-treated MSCs (MSCs+IFN- $\gamma$ ) were detected by flow cytometry using monoclonal antibodies specific for HLA-I, HLA-DR, CD80, and CD86.

Statistical analysis. The analysis was conducted with SPSS 19.0 software (IBM Corp., Armonk, NY, USA), and the data are expressed as the mean \pm standard deviation. Statistical comparisons between two groups were performed by the t-test, and a one-way analysis of variance followed by Tukey's test were used for comparisons among multiple groups. $\mathrm{P}<0.05$ was considered to indicate a statistically significant difference. All experiments were repeated at least three times as previously described $(24,25)$.

\section{Results}

Biological characteristics of MSCs. Cell surface marker analysis by flow cytometry showed that all four types of MSCs positively expressed MSC markers, including vimentin, CD90, and CD105, and they also positively expressed nestin and NANOG, but they showed no expression of hematopoietic stem cell marker CD34 and epithelial cell marker CK19 (Fig. 1).

Colony-forming efficiency assays were used to examine the self-renewal capacity of MSCs. All four MSCs at passage 3 seeded in 6 -well plates at $1 \times 10^{2}$ cells/well for 14 days can form colonies. GMSCs exhibited highest self-renewal capacity, UCMSC displayed the lowest colony formation units, between SHED and BMSC there were no significant differences in colony-forming efficiency (Fig. 2).

To investigate the differentiation potential of the MSCs, cells were induced to osteogenic, adipogenic, and chondrogenic differentiation. Osteogenic differentiation was observed with Alizarin Red staining, and calcium deposition was seen in all four MSCs. Adipogenic differentiation was verified by Oil Red O staining, and the accumulation of cytoplasmic lipid vacuoles was distinctly observed. Chondrogenic differentiation was verified by Alcian Blue staining and was demonstrated in all tested cells (Fig. 3). The results indicated successful differentiation of MSCs into osteogenic, adipogenic, and chondrogenic lineages. 

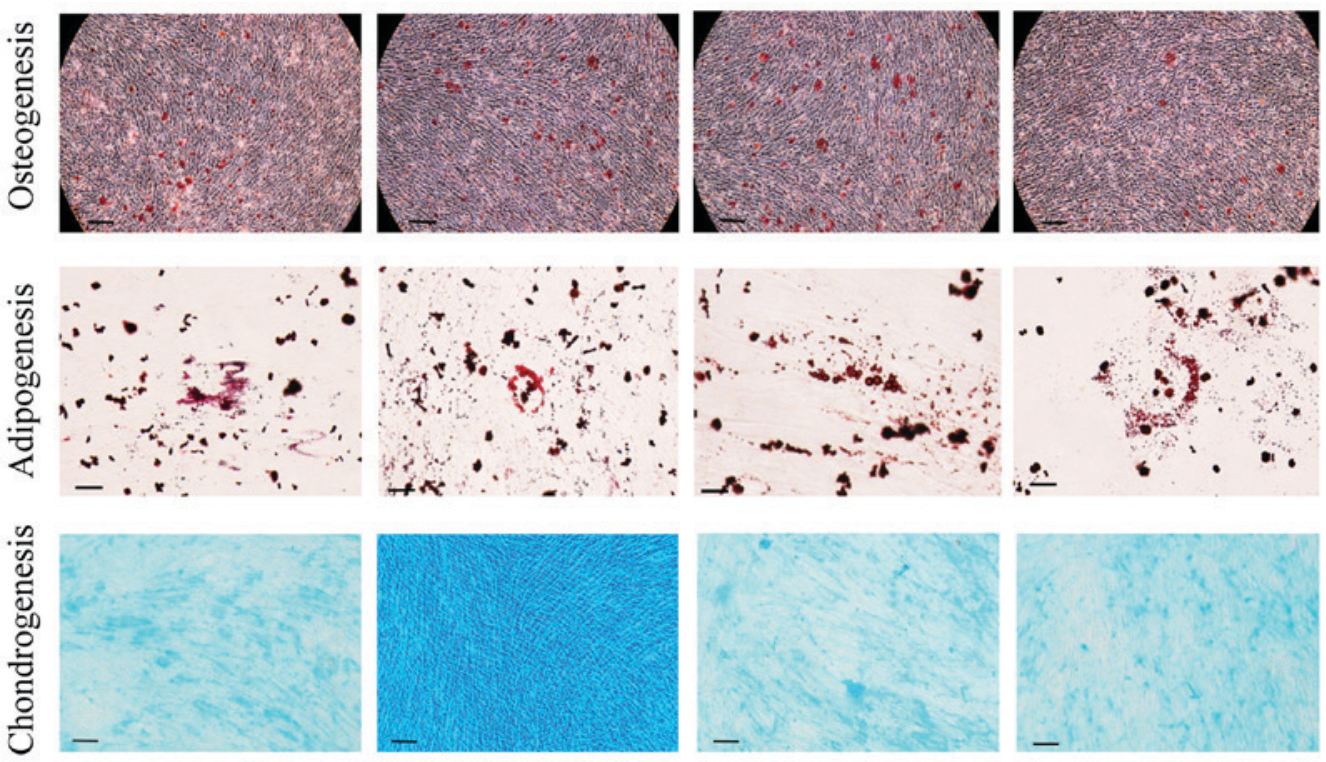

SHED

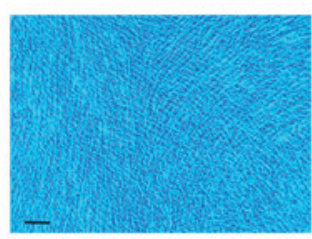

GMSC

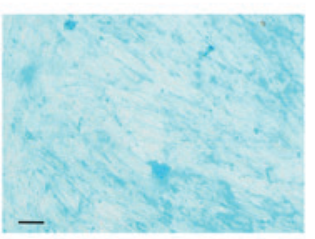

BMSC

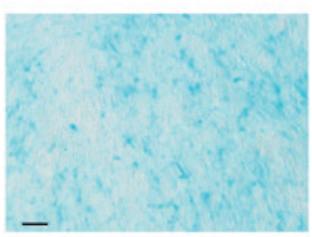

UCMSC

Figure 3. Osteogenic, adipogenic and chondrogenic differentiation of MSCs. MSCs were induced to differentiate toward osteogenic, adipogenic and chondrogenic lineages as verified by Alizarin Red, Oil Red O and Alcian Blue staining, respectively (osteogenic and chondrogenic differentiation: scale bar, $100 \mu$ m; adipogenic differentiation: scale bar, $50 \mu \mathrm{m}$ ). MSCs, mesenchymal stem cells; SHEDs, stem cells from human exfoliated deciduous teeth; UCMSCs, umbilical cord-derived mesenchymal stem cells; GMSCs, gingiva-derived mesenchymal stem cells; BMSCs, bone marrow mesenchymal stem cells.

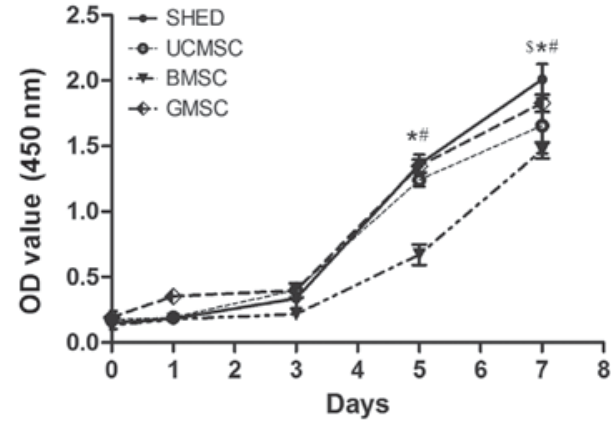

Figure 4. Comparison of proliferation ability in MSCs. Cell Counting kit- 8 assay revealed that the relative OD values for SHEDs were significantly higher than those for the other three types of MSCs at 5 and 7 days after seeding. Results represent the mean \pm standard deviation of five independent experiments. ${ }^{*} \mathrm{P}<0.05$ vs. BMSC; ${ }^{\#} \mathrm{P}<0.05$ vs. UCMSC; ${ }^{\$} \mathrm{P}<0.05$ vs. GMSC OD, optical density; SHEDs, stem cells from human exfoliated deciduous teeth; UCMSCs, umbilical cord-derived mesenchymal stem cells; GMSCs, gingiva-derived mesenchymal stem cells; BMSCs, bone marrow mesenchymal stem cells.

Proliferation capacity of MSCs. Cell proliferation was monitored over a period of 7 days post-seeding (Fig. 4). The proportion of all MSCs increased markedly from the third day of proliferation, and we found that SHED showed enhanced proliferation capacity compared to the other MSCs. On the fifth day, except for between the SHED and GMSC groups, and the GMSC and UCMSC groups, there were significant differences among the other groups $(\mathrm{P}<0.05)$. On the seventh day, except for between the GMSC and UCMSC groups, there were significant differences among the other groups $(\mathrm{P}<0.05)$.

Tumorigenicity of MSCs. To compare colony formation of the four types of MSCs in vitro, we performed soft agar colony assays using $1 \times 10^{4}$ cells/well in a 6 -well plate, and HeLa cells were used as a positive control. After being cultured for 21 days, none of the four MSC groups formed colonies, but the HeLa cell group formed a large number of colonies (Fig. 5A). HeLa cells, but not the four types of MSCs, showed anchorage-independent cell growth in soft agar. MSCs showed a notably lower ability to form colonies than HeLa cells in vitro. Subcutaneous inoculation of MSCs into nude mice showed that none of the four types of MSCs caused any clinical signs during the 6-month experimental period, and there was no tumor formation in any mice at the injection area and around that area, but abnormal masses at injection areas were found in the HeLa cell group (Fig. 5B). In the HeLa cell group, 2 weeks after subcutaneous inoculation abnormal masses at injection areas can be seen. Continuous observation for 2 months, the maximum tumor size was $1,884 \mathrm{~mm}^{3}$, no multiple tumors were observed.

There were no significant structural chromosomal abnormalities/aberrations in the karyotypes of any diploid cells, indicating that the karyotypes were stable.

Immunogenicity of MSCs. To investigate whether allogeneic human MSCs can stimulate the proliferation of PBMCs, PBMCs were co-cultured with MSCs that were pretreated with mitomycin C. As shown in Fig. 6A, after a mixed co-culture for 3 days, phytohemagglutinin (PHA) induced a very strong proliferative response in allogeneic lymphocytes $(\mathrm{P}<0.01)$, but none of the allogeneic MSCs elicited any proliferative response compared to the control group; MSCs inhibited the proliferation of lymphocytes, and all MSC groups showed a significant difference from the control group $(\mathrm{P}<0.01)$. Comparing the four types of MSCs, the BMSC and UCMSC groups $(\mathrm{P}<0.05)$ and the GMSC and UCMSC groups $(\mathrm{P}<0.05)$ showed a significant difference, but there was no significant difference between the other MSC groups. 

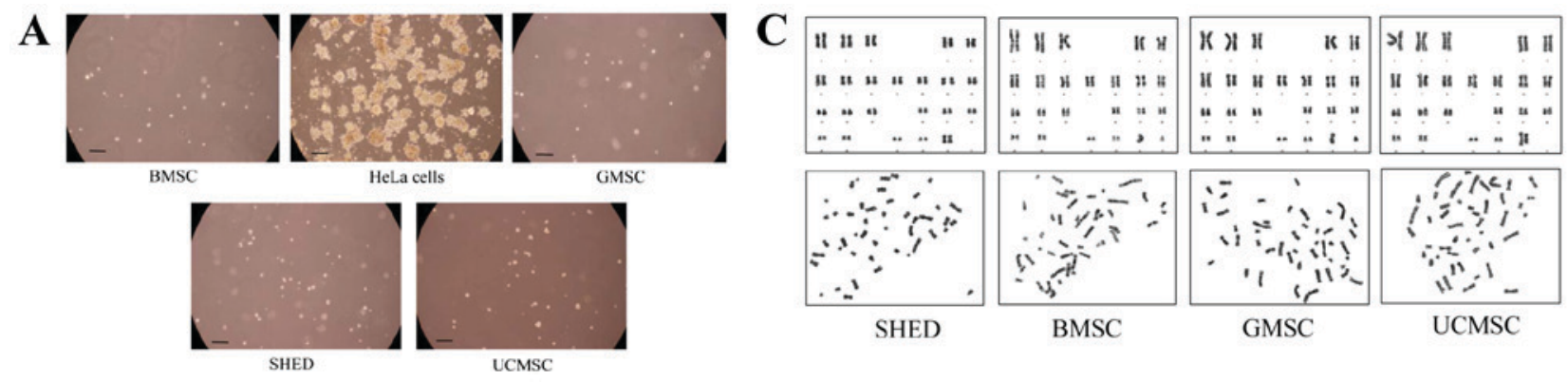

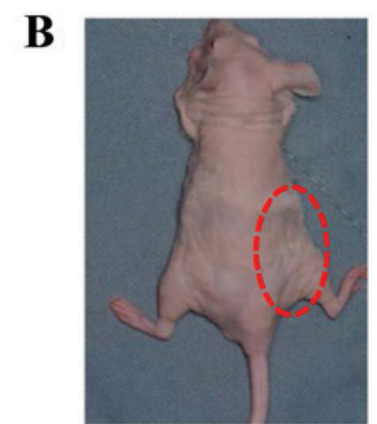

BMSC

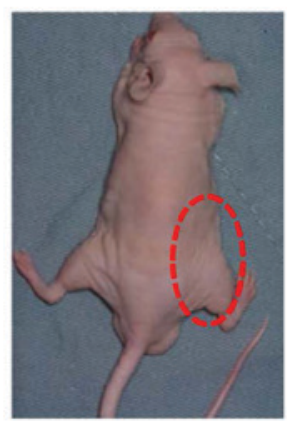

UCMSC

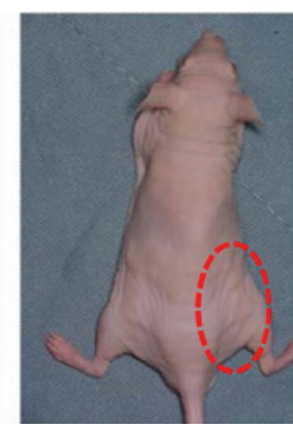

GMSC

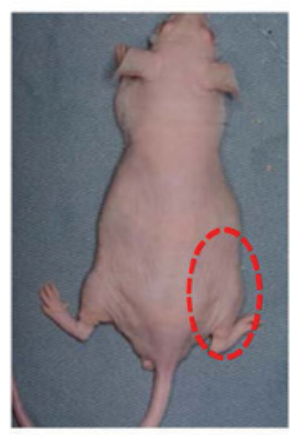

SHED

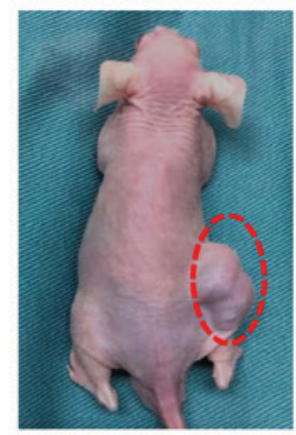

HeLa cells

Figure 5. Tumorigenicity of MSCs. (A) Soft agar colony assay for MSCs and HeLa cells. Representative images of colonies in the soft agar assay for BMSCs, GMSCs, SHEDs, UCMSCs and HeLa cells. A large number of colonies were observed in the HeLa group, but no colonies were identified in the MSC groups (scale bar, $200 \mu \mathrm{m}$ ). (B) BMSCs, UCMSCs, GMSCs, SHEDs and HeLa cells were transplanted into nude mice. Following 2 months, tumors were identified in the injection areas of the group transplanted with HeLa cells, but at 6 months, no tumors were observed in the other groups transplanted with stem cells. (C) Karyotype analysis of SHEDs, BMSCs, GMSCs and UCMSCs. The upper panel shows G-bands and the lower panel shows natural distribution. No karyotype abnormalities were identified for any cells. SHEDs, stem cells from human exfoliated deciduous teeth; UCMSCs, umbilical cord-derived mesenchymal stem cells; GMSCs, gingiva-derived mesenchymal stem cells; BMSCs, bone marrow mesenchymal stem cells.

The results of flow cytometry revealed that all four types of MSCs expressed HLA-I, but they did not express HLA-DR or the costimulatory molecules CD80 and CD86. After treatment with IFN- $\gamma$ for $72 \mathrm{~h}$, the expressions of HLA-I, CD80, and CD86 showed no obvious change in the MSCs, but the expression of HLA-DR was significantly upregulated in BMSCs only (Fig. 6B-I).

\section{Discussion}

MSCs have been widely used in vivo and in vitro because of their strong self-renewal, proliferation, multilineage differentiation, and immunomodulatory abilities. MSCs from different sources may differ in their biological characteristics. Understanding the differences in biological characteristics of MSCs from different sources can guide the selection of more suitable cell sources in clinical applications given different treatment requirements and is helpful for selecting safer and more efficient seed cells in clinical treatment and scientific research. In this study, we investigated the differences in biological characteristics, including surface markers, colony-forming efficiency, multi-potent differentiation, proliferation capacity, tumorigenicity, and immunogenicity among these human MSCs from different origins, and we advanced our understanding of the advantages of each cell.

It has been found that MSCs have similar phenotypes, and the expression of MSC markers associated with stress or aging remains unchanged (26). All four types of MSCs in this study positively expressed MSC surface markers, including vimentin, CD105, and CD90, and they did not express the hematopoietic stem cell marker CD34 or the epithelial cell marker CK19. Nestin and NANOG are markers of embryonic stem cells, and they play an important role in the maintenance of pluripotency and self-renewal (27). All four types of MSCs positively expressed nestin and NANOG, which indicates that the four types of MSCs maintained pluripotency and have good self-renewal ability.

Self-renewal and multi-potent differentiation are two important properties of MSCs. All four types of MSCs in this study had good colony-forming efficiency and could be induced to differentiate into osteogenic, adipogenic, and chondrogenic lineages. It has been suggested that the MSC cell type should be selected depending on the regenerative treatment regimen (28). In addition, the proliferation capacity of stem cells is also an important factor in stem cell therapies. SHEDs and UCMSCs have shown a higher proliferation capacity in comparison with BMSCs in previous studies $(13,26)$. Our study showed that the strongest proliferative ability was found in SHEDs, followed by GMSCs and UCMSCs, and the lowest proliferative ability was found in BMSCs; these results are consistent with previous studies by other groups. SHEDs are derived from the dental pulp tissue of deciduous teeth, an immature tissue. The proliferative capacity of SHEDs is relatively strong since they are in an active state and show high telomerase activity when deciduous teeth are replaced by permanent teeth (22).

As UCMSCs are separated from the fetal umbilical cord, a degenerative tissue, they exhibit less proliferative capacity than SHEDs. GMSCs have a higher proliferative capacity than BMSCs, and this may be related to their active state and fast oral metabolism. 

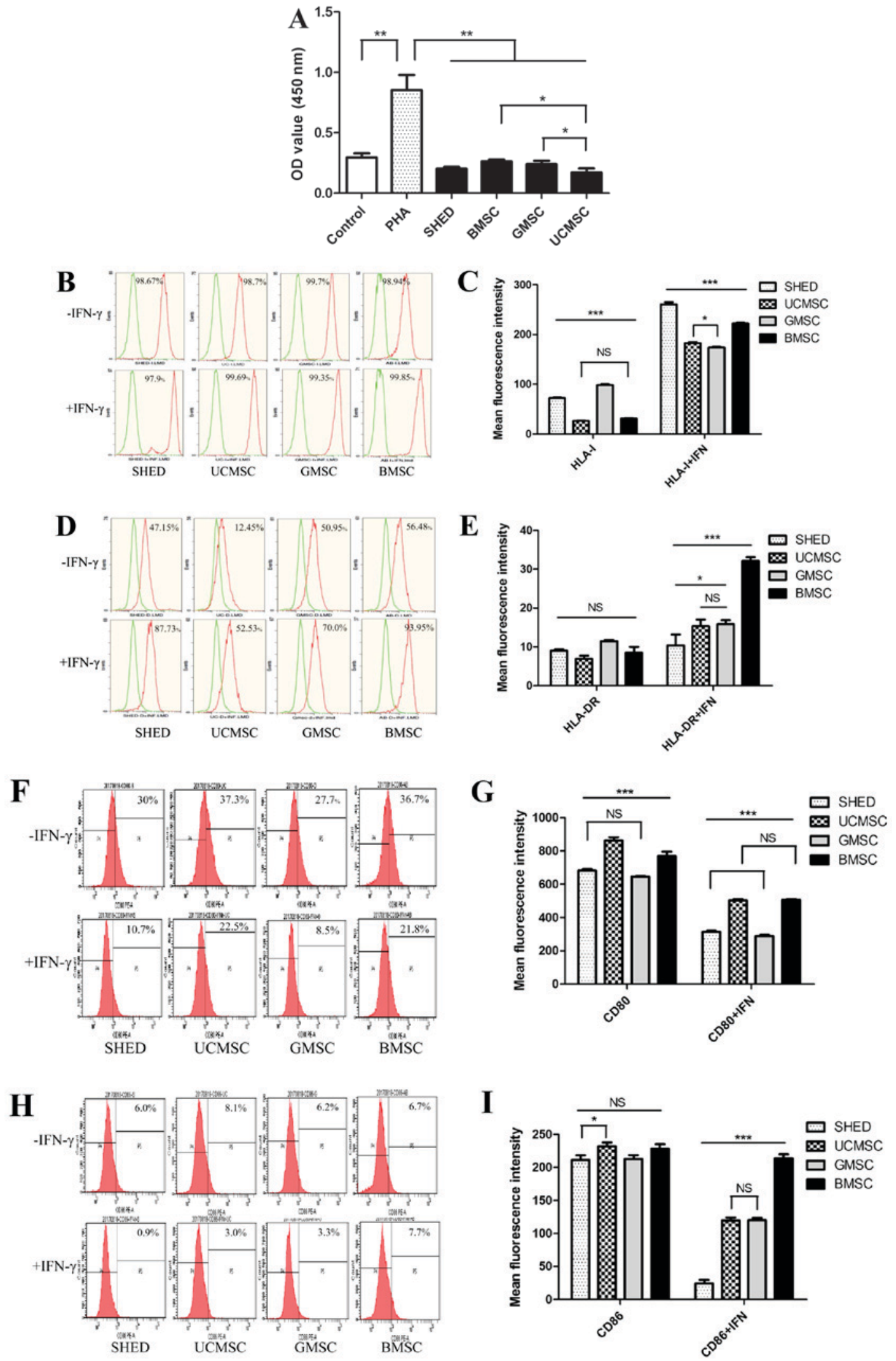

Figure 6. Immunogenicity characterization of MSCs. (A) The proliferation capacity of peripheral blood mononuclear cells following mixed co-culture for 3 days. Cell Counting kit- 8 assay revealed that the relative OD values for the four types of MSCs were significantly lower than those in the control and PHA groups. When comparing the four types of MSCs, the OD value for UCMSCs was lower than that of BMSCs and GMSCs. Results represent the mean \pm standard deviation of five independent experiments. (B and C) All MSCs exhibited high expression of HLA-I prior to or following IFN- $\gamma$ treatment. (D and E) All MSCs had a lower expression of HLA-DR prior to IFN- $\gamma$ treatment, but following IFN- $\gamma$ treatment, the expression of HLA-DR was significantly upregulated. (F and G) The expression of CD80 was very low prior to and following stimulation with IFN- $\gamma$. (H and I) The expression of CD86 was very low prior to and following stimulation with IFN- $\gamma$. Results represent the mean \pm standard deviation of three independent experiments. ${ }^{*} \mathrm{P}<0.05$, ${ }^{* *} \mathrm{P}<0.01$ and ${ }^{* * * *} \mathrm{P}<0.001$, as indicated. NS, not significant; MSCs, mesenchymal stem cells; OD, optical density; PHA, phytohemagglutinin; SHEDs, stem cells from human exfoliated deciduous teeth; UCMSCs, umbilical cord-derived mesenchymal stem cells; GMSCs, gingiva-derived mesenchymal stem cells; BMSCs, bone marrow mesenchymal stem cells; HLA, human leukocyte antigen; -DR, antigen D related; IFN- $\gamma$, interferon- $\gamma$; CD, cluster of differentiation. 
The evaluation of bio-safety is important for clinical therapies utilizing MSCs, including external safety and intrinsic safety tests. The external safety tests include pathogen detection, mycoplasma detection, the asepsis test, and the endotoxin test, while intrinsic safety tests include acute and chronic toxicities, tumorigenicity, immunogenicity, and chromosome aberrations.

In this study, stem cells at passages 3 to 5 were used for experiments; these cells are relatively young, and their performance is relatively stable. Cell clones were not formed in vitro under soft agar culture in any of the four types of MSCs. After in vivo transplantation into nude mice, the stem cells did not form any abnormal tumor tissues for up to 6 months, and karyotype analysis showed that no karyotypic abnormality was found in any of the four types of MSCs, thus indicating the safety of all four. These results are consistent with those obtained by other groups $(26,29)$.

MSCs have been demonstrated to have some immunomodulatory functions in vitro, such as direct suppression of allogeneic and mitogenic T-cell proliferation (30). BMSCs have been implicated as a potentially feasible treatment approach for several diseases, such as graft-versus-host disease (31) and autoimmune diseases (32). Due to their immunomodulatory properties, SHEDs are an accessible and feasible MSC source for treating immune disorders like systemic lupus erythematosus (22). The wide usefulness of MSCs can be attributed to their low immunogenicity and immunomodulatory functions. When MSCs were co-cultured with PBMCs under cell-cell contact conditions, none of the allogeneic MSCs elicited a proliferative response in PBMCs, indicating that the four types of MSCs displayed low immunogenicity. This result is consistent with the notion that the cell-cell contact mechanism may partly contribute to MSC-mediated suppression of PBMC proliferation $(16,25,33)$. UCMSCs are isolated from fetal tissue, and as they are more primitive than adult stem cells, they showed higher immunomodulatory ability, but the immunomodulatory ability of BMSCs and GMSCs showed no significant difference in this study. This result is not completely consistent with previous studies $(16,26)$.

To further understand the mechanism of low immunogenicity of the four types of MSCs, we analyzed the expression of HLA and costimulatory molecules (CD80 and CD86). It is well known that T-lymphocyte activation requires two signals, the T-cell receptors with antigenic peptides presented by HLA-DR molecules and the costimulatory molecules CD80 and CD86 (34). HLA-I is expressed on all nucleated cell surfaces, which are mainly responsible for the presentation of endogenous antigens, whereas HLA-DR is expressed only on the surface of antigen-presenting cells, which are mainly responsible for the presentation of exogenous antigens. However, recent studies have found that BMSCs also express HLA-DR, and its expression level is affected by individual differences and the cell microenvironment (35). All four types of MSCs expressed a high level of HLA-I, but HLA-DR expression was very low under resting conditions. However, the expression of HLA-DR was upregulated in the four types of MSCs, especially in BMSCs, after stimulation with IFN- $\gamma$, suggesting that BMSCs exhibited the best antigen-presenting potential. Also, the expression of costimulatory molecules
CD80 and CD86 in all four types of MSCs was low either under resting conditions or under stimulation with IFN- $\gamma$.

In conclusion, the populations of MSCs derived from different sources exhibited variability in their proliferative capacity and immunomodulatory ability, although they displayed similar phenotypes. Based on these results, in which SHEDs and GMSCs showed a higher proliferation capacity, we conclude that SHEDs and GMSCs are perhaps suited for tissue regeneration-related cellular therapies. UCMSCs showed higher immunomodulatory ability, so they are better suited for cellular therapy for some immune-related diseases. BMSCs can more easily induce immune reactions than the other three MSCs in the host after cell transplantation. These data will provide helpful information for the clinical application of MSCs.

\section{Acknowledgements}

The authors would like to thank the Oral Stem Cell Bank of Beijing, Tason Biotech Co., Ltd. for their assistance in stem cell collection.

\section{Funding}

The present study was supported by the National Natural Science Fund Emergency Management Project (grant no. 81541110), the Tason Oral Medicine Development Fund (grant no. 2016.7-2019.12), and the International Scientific and Technological Cooperation and Exchange (grant no. 2014DFA31520).

\section{Availability of data and materials}

The datasets used and/or analyzed during the current study are available from the corresponding author on reasonable request.

\section{Authors' contributions}

L-HG and B-HX designed the present study. JL and S-QX performed the experiments and analyzed the data. SY contributed to sample collection, acquisition and data analysis. Y-MZ contributed to animal study and data analysis. JL and Y-MZ drafted the manuscript and revised it critically for important intellectual content. All authors read and approved the final manuscript, and agreed to be accountable for all aspects of the study.

\section{Ethics approval and consent to participate}

The present study was approved by the Institutional Review Board of Peking University School and Hospital of Stomatology (no. PKUSSIRB-201734036); written informed consent was obtained from all participants. The animal studies were approved by the Animal Ethics Committee of the China-Japan Friendship Hospital (no. 170103).

\section{Patient consent for publication}

Written informed consent was obtained from all participants. 


\section{Competing interests}

The authors declare that they have no competing interests.

\section{References}

1. Prockop DJ: Marrow stromal cels as stem cells for nonhematopoietic tissues. Science 276: 71-74, 1997.

2. Pittenger MF, Mackay AM, Beck SC, Jaiswal RK, Douglas R, Mosca JD, Moorman MA, Simonetti DW, Craig S and Marshak DR: Multilineage potential of adult human mesenchymal stem cells. Science 284: 143-147, 1999.

3. Pittenger MF and Martin BJ: Mesenchymal stem cells and their potential as cardiac therapeutics. Circ Res 95: 9-20, 2004.

4. Satake K, Lou J and Lenke LG: Migration of mesenchymal stem cells through cerebrospinal fluid into injured spinal cord tissue. Spine (Phila Pa 1976) 29: 1971-1979, 2004.

5. Satija NK, Singh VK, Verma YK, Gupta P, Sharma S, Afrin F, Sharma M, Sharma P, Tripathi RP and Gurudutta GU: Mesenchymal stem cell-based therapy: A new paradigm in regenerative medicine. J Cell Mol Med 13: 4385-4402, 2009.

6. Taran R, Mamidi MK, Singh G, Dutta S, Parhar IS, John JP, Bhonde R, Pal R and Das AK: In vitro and in vivo neurogenic potential of mesenchymal stem cells isolated from different sources. J Biosci 39: 157-169, 2014.

7. Ma J, Both SK, Yang F, Cui FZ, Pan J, Meijer GJ, Jansen JA and van den Beucken JJ: Concise review: Cell-based strategies in bone tissue engineering and regenerative medicine. Stem Cells Transl Med 3: 98-107, 2014.

8. Shang Q, Wang Z, Liu W, Shi Y, Cui L and Cao Y: Tissue-engineered bone repair of sheep cranial defects with autologous bone marrow stromal cells. J Craniofac Surg 12: 586-595, 2001

9. Ferrari G, Cusella-De Angelis G, Coletta M, Paolucci E, Stornaiuolo A, Cossu G and Mavilio F: Muscle regeneration by bone marrow-derived myogenic progenitors. Science 279 : 1528-1530, 1998.

10. Shintani S, Murohara T, Ikeda H, Ueno T, Honma T, Katoh A, Sasaki K, Shimada T, Oike Y and Imaizumi T: Mobilization of endothelial progenitor cells in patients with acute myocardial infarction. Circulation 103: 2776-2779, 2001.

11. Ringdén O, Uzunel M, Rasmusson I, Remberger M, Sundberg B, Lönnies H, Marschall HU, Dlugosz A, Szakos A, Hassan Z, et al Mesenchymal stem cells for treatment of therapy-resistant graft-versus-host disease. Transplantation 81: 1390-1397, 2006.

12. Angelopoulou M, Novelli E, Grove JE, Rinder HM, Civin C, Cheng L and Krause DS: Cotransplantation of human mesenchymal stem cells enhances human myelopoiesis and megakaryocytopoiesis in NOD/SCID mice. Exp Hematol 31: 413-420, 2003

13. Miura M, Gronthos S, Zhao M, Lu B, Fisher LW, Robey PG and Shi S: SHED: Stem cells from human exfoliated deciduous teeth. Proc Natl Acad Sci USA 100: 5807-5812, 2003.

14. Ren H, Sang Y, Zhang F, Liu Z, Qi N and Chen Y: Comparative analysis of human mesenchymal stem cells from umbilical cord dental pulp, and menstrual blood as sources for cell therapy. Stem Cells Int 2016: 3516574, 2016.

15. Ren HY, Zhao QJ, Xing W, Yang SG, Lu SH, Ren Q, Zhang L and Han ZC: Differentiation of human umbilical cord derived mesenchymal stem cells into low immunogenic and functional hepatocyte-like cells in vitro. Zhongguo Yi Xue Ke Xue Yuan Xue Bao 32: 190-194, 2010 (In Chinese)

16. Zhang Q, Shi S, Liu Y, Uyanne J, Shi Y, Shi S and Le AD: Mesenchymal stem cells derived from human gingiva are capable of immunomodulatory functions and ameliorate inflammation-related tissue destruction in experimental colitis. J Immunol 183: 7787-7798, 2009.

17. Tomar GB, Srivastava RK, Gupta N, Barhanpurkar AP, Pote ST, Jhaveri HM, Mishra GC and Wani MR: Human gingiva-derived mesenchymal stem cells are superior to bone marrow-derived mesenchymal stem cells for cell therapy in regenerative medicine. Biochem Biophys Res Commun 393: $377-383,2010$.
18. Wang F, Yu M, Yan X, Wen Y, Zeng Q, Yue W, Yang P and Pei X: Gingiva-derived mesenchymal stem cell-mediated therapeutic approach for bone tissue regeneration. Stem Cells Dev 20: 2093-2102, 2011

19. Jacobs SA, Roobrouck VD, Verfaillie CM and Van Gool SW: Immunological characteristics of human mesenchymal stem cells and multipotent adult progenitor cells. Immunol Cell Biol 91: 32-39, 2013.

20. Deans RJ and Moseley AB: Mesenchymal stem cells: Biology and potential clinical uses. Exp Hematol 28: 875-884, 2000.

21. Inoue S, Popp FC, Koehl GE, Piso P, Schlitt HJ, Geissler EK and Dahlke MH: Immunomodulatory effects of mesenchymal stem cells in a rat organ transplant model. Transplantation 81: 1589-1595, 2006.

22. Yamaza T, Kentaro A, Chen C, Liu Y, Shi Y, Gronthos S, Wang S and Shi S: Immunomodulatory properties of stem cells from human exfoliated deciduous teeth. Stem Cell Res Ther 1: $5,2010$.

23. Li P,Zhao Y and Ge L: Therapeutic effects of human gingiva-derived mesenchymal stromal cells on murine contact hypersensitivity via prostaglandin E2-EP3 signaling. Stem Cell Res Ther 7: 103, 2016.

24. Fu X, Chen Y, Xie FN, Dong P, Liu WB, Cao Y, Zhang WJ and Xiao R: Comparison of immunological characteristics of mesenchymal stem cells derived from human embryonic stem cells and bone marrow. Tissue Eng Part A 21: 616-626, 2015.

25. Wang M, Yang Y, Yang D, Luo F, Liang W, Guo S and Xu J: The immunomodulatory activity of human umbilical cord blood-derived mesenchymal stem cells in vitro. Immunology 126: 220-232, 2009.

26. de Witte SFH, Lambert EE, Merino A, Strini T, Douben HJCW, O'Flynn L, Elliman SJ, de Klein AJEMM, Newsome PN, Baan CC and Hoogduijn MJ: Aging of bone marrow- and umbilical cord-derived mesenchymal stromal cells during expansion. Cytotherapy 19: 798-807, 2017.

27. Luo W, Li S, Peng B, Ye Y, Deng X and Yao K: Embryonic stem cells markers SOX2, OCT4 and Nanog expression and their correlations with epithelial-mesenchymal transition in nasopharyngeal carcinoma. PLoS One 8: e56324, 2013.

28. Isobe $Y$, Koyama N, Nakao K, Osawa K, Ikeno M, Yamanaka S, Okubo Y, Fujimura K and Bessho K: Comparison of human mesenchymal stem cells derived from bone marrow, synovial fluid, adult dental pulp, and exfoliated deciduous tooth pulp. Int J Oral Maxillofac Surg 45: 124-131, 2016.

29. Nakashima M, Iohara K, Murakami M, Nakamura H, Sato $Y$, Ariji Y and Matsushita K: Pulp regeneration by transplantation of dental pulp stem cells in pulpitis: A pilot clinical study. Stem Cell Res Ther 8: 61, 2017.

30. Bartholomew A, Sturgeon C, Siatskas M, Ferrer K, McIntosh K, Patil S, Hardy W, Devine S, Ucker D, Deans R, et al: Mesenchymal stem cells suppress lymphocyte proliferation in vitro and prolong skin graft survival in vivo. Exp Hematol 30: 42-48, 2002.

31. Le Blanc K, Rasmusson I, Sundberg B, Götherström C, Hassan M, Uzunel M and Ringdén O: Treatment of severe acute graft-versus-host disease with third party haploidentical mesenchymal stem cells. Lancet 363: 1439-1441, 2004.

32. Sun L, Akiyama K, Zhang H, Yamaza T, Hou Y, Zhao S, Xu T, Le A and Shi S: Mesenchymal stem cell transplantation reverses multiorgan dysfunction in systemic lupus erythematosus mice and humans. Stem Cells 27: 1421-1432, 2009.

33. Sheng H, Wang Y, Jin Y, Zhang Q, Zhang Y, Wang L, Shen B, Yin S, Liu W, Cui L and Li N: A critical role of IFNgamma in priming MSC-mediated suppression of $\mathrm{T}$ cell proliferation through up-regulation of B7-H1. Cell Res 18: 846-857, 2008.

34. Ryan JM, Barry FP, Murphy JM and Mahon BP: Mesenchymal stem cells avoid allogeneic rejection. J Inflamm (Lond) 2: 8, 2005.

35. Bocelli-Tyndall C, Zajac P, Di Maggio N, Trella E, Benvenuto F, Iezzi G, Scherberich A, Barbero A, Schaeren S, Pistoia V, et al: Fibroblast growth factor 2 and platelet-derived growth factor, but not platelet lysate, induce proliferation-dependent, functional class II major histocompatibility complex antigen in human mesenchymal stem cells. Arthritis Rheum 62: 3815-3825, 2010.

This work is licensed under a Creative Commons Attribution-NonCommercial-NoDerivatives 4.0 International (CC BY-NC-ND 4.0) License. 